\title{
Influence of UGTIAI *6/*28 Polymorphisms on Irinotecan-Related Toxicity and Survival in Pediatric Patients with Relapsed/Refractory Solid Tumors Treated with the VIT Regimen
}

This article was published in the following Dove Press journal:

Pharmacogenomics and Personalized Medicine

\author{
Xiaoqin Zhu $\mathbb{D}^{1,2, *}$ \\ Jia Zhu $\mathbb{D}^{1,2, *}$ \\ Feifei Sun (D) ${ }^{1,2, *}$ \\ Zijun Zhen (iD) ${ }^{1,2}$ \\ Dalei Zhou ${ }^{1,3}$ \\ Suying $\mathrm{Lu}^{\mathrm{I}, 2}$ \\ Junting Huang $\mathbb{D}^{1,2}$ \\ Yi Que (D) ${ }^{1,2}$ \\ Lian Zhang ${ }^{1,2}$ \\ Ruiqing Cai ${ }^{1,2}$ \\ Juan Wang ${ }^{1,2}$ \\ Yizhuo Zhang ${ }^{1,2}$
}

'State Key Laboratory of Oncology in South China, Collaborative Innovation Center for Cancer Medicine, Sun Yat-Sen University Cancer Center, Guangzhou, People's Republic of China; ${ }^{2}$ Department of Pediatric Oncology, Sun Yat-Sen University Cancer Center, Guangzhou, People's Republic of China; ${ }^{3}$ Department of Molecular Diagnostics, Sun Yat-Sen University Cancer Center, Guangzhou, People's Republic of China

*These authors contributed equally to this work
Correspondence: Yizhuo Zhang; Juan

Wang

Email zhangyzh@sysucc.org.cn;

wangjuan@sysucc.org.cn

\begin{abstract}
Objective: The association between UGT1A $1 * 6 / * 28$ polymorphisms and treatment outcomes of irinotecan in children remains unknown. This retrospective study investigated the influence of UGT1A $1 * 6 / * 28$ polymorphisms on irinotecan toxicity and survival of pediatric patients with relapsed/refractory solid tumors.

Methods: The present study enrolled a total of 44 patients aged younger than 18 years at Sun Yat-sen University Cancer Center between 2014 and 2017.

Results: There were 26 boys and 18 girls; the median age at first VIT course was six years (range: 1-18 years). The tumor types included neuroblastoma $(\mathrm{n}=25)$, rhabdomyosarcoma $(\mathrm{n}=11)$, Wilm's tumor $(\mathrm{n}=4)$, medulloblastoma $(\mathrm{n}=2)$, and desmoplastic small round cell tumor $(\mathrm{n}=2)$. Overall, 203 courses of VIT regimens were prescribed. Neither UGT1A1*6 nor $* 28$ polymorphisms were associated with the incidence rates of severe (grade III-IV) irinotecan-related toxicities, but tended to reduce the patient overall survival (UGT1A1*6, $P=0.146$; UGT1A $1 * 28, P=0.195)$. Moreover, patients with mutant UGT1A $1 * 6$ genotypes were more likely to develop grade I-IV irinotecan-related diarrhea $(P=0.043)$ and anemia $(P=0.002)$. Overall, the UGT1A $1 * 28$ polymorphism may play a protective role against irinotecan-related diarrhea and abdominal pain.

Conclusion: In relapsed/refractory pediatric solid tumors, the UGT1A $1 * 6$ polymorphism was a risk factor of irinotecan-related diarrhea and anemia. The UGT1A1*28 polymorphism may serve a protective role in irinotecan-related abdominal pain and diarrhea. Both mutations had a tendency to be risk factors for survival. Nevertheless, prospective studies are required to verify such conclusions.
\end{abstract}

Keywords: UGT1A1 $* 6 / * 28$ polymorphism, irinotecan toxicity, pediatric, relapsed/ refractory solid tumors

\section{Introduction}

Irinotecan, a semisynthetic water-soluble analog of camptothecin, is a prodrug activated by carboxylesterases to produce $\mathrm{SN}-38$ in the human liver, intestinal mucosa, and plasma. ${ }^{1-3}$ Irinotecan has been used clinically as a single agent or in combination with other chemotherapeutic agents for the treatment of lung, colorectal (CRC), esophageal, gastric, cervical, ovarian, and gynecological cancers. ${ }^{2,4-6}$ Irinotecan has also shown notable antitumor activity in many recurrent and newly diagnosed pediatric solid tumors, including neuroblastoma (NB), rhabdomyosarcoma (RMS), brain tumors, non- 
Hodgkin lymphoma, and hepatoblastoma. ${ }^{5,7}$ Moreover, a variety of schedules have been used in phase $\mathrm{I}^{7-10}$ or phase II $^{1,3,11}$ studies of irinotecan in children; however, up to $36 \%$ of patients experience severe, potentially lifethreatening toxicities (ie, diarrhea) following irinotecan treatment, depending on the irinotecan dosage and regimen. ${ }^{2,4,7,10,12}$ These toxicities can cause unexpected reductions in the dosage or treatment withdrawal, ${ }^{4}$ which has been shown to be associated with increased disease progression and lower survival in patients with colorectal cancer. $^{13}$

Both irinotecan and SN-38 have inhibitory effects on topoisomerase I, which breaks DNA strands during the replicative phase in main toxicity-targeted tissues and tumors, ${ }^{14}$ which leads to gastrointestinal (ie, diarrhea) or hematological toxicities (ie, neutropenia and leucopenia). SN-38 has a substantially greater potency to produce such lesions compared to that of irinotecan. ${ }^{14-16}$ However, the accumulation of SN-38 in the intestine can result in direct enteric injury, which has been shown to be responsible for the diarrhea attributed to irinotecan administration., ${ }^{2,6,15}$ A large body of evidence suggests that genetic differences may play a key role in drug pharmacokinetics and pharmacodynamics, which is particularly crucial when a drug is metabolized by a specific predominant route, without many other alternative pathways. This is the case of irinotecan, for which SN-38 metabolism is largely reliant on the uridine diphosphate glucuronosyltransferase (UGT) enzyme family. ${ }^{6,12}$ In the liver, SN-38 is conjugated to glucuronic acid by UDPglycosyltransferase 1 polypeptide A1 (UGT1A1) to form $\mathrm{SN}-38 \mathrm{G},{ }^{2,5,14}$ which represents the major elimination pathway of SN-38. ${ }^{15}$ Therefore, the UGT1A1-mediated glucuronidation of $\mathrm{SN}-38$ to $\mathrm{SN}-38 \mathrm{G}$ may play a key role in protection against irinotecan-related toxicities; ${ }^{2}$ however, UGT1A1 genetic polymorphisms have been reported to be related with decreased SN-38 glucuronidation, ${ }^{2,17}$ and will therefore be likely to play a significant role in the pharmacokinetic variability of irinotecan. ${ }^{18}$ The pharmacogenetics of irinotecan has been subjected to considerable study to optimize therapy through personalized medicine and to reduce any adverse events. ${ }^{4}$ The relevance of the decreased glucuronidation of SN-38 for irinotecan toxicity was first shown in patients with Gilbert syndrome, ${ }^{19}$ leading to the identification of UGT1A1 polymorphisms as the main predictors of irinotecan toxicity in cancer patients. ${ }^{4,14,19}$

The two most extensively reported single-nucleotide polymorphisms (SNPs) of UGT1A1 concerning irinotecan toxicity are the $* 6$ variant (rs4148323) ${ }^{17}$ and $* 28$ variant (rs8175347), ${ }^{6,12}$ focusing on adult solid tumors; ${ }^{16,17,20-22}$ however, little is known about the relationship between UGT1A $1 * 6 / * 28$ polymorphisms and irinotecan treatment outcomes in pediatric solid tumors. Therefore, this retrospective study aimed to evaluate the influence of UGT1A1 $* 6 / * 28$ polymorphisms on irinotecan-related toxicities and survival in children with relapsed/refractory solid tumors who received irinotecan-containing VIT regimens.

\section{Methods}

\section{Patients and Treatment}

We searched the Sun Yat-sen University Cancer Center (SYSUCC) database for patient data that met the requisite inclusion criteria". The inclusion criteria consisted of 1) age $\leq 18$ years; 2 ) diagnosis of relapsed/refractory solid tumor; 3 ) genotype for the UGT1A1 $* 6 / * 28$ polymorphism; and 4) administration of VIT chemotherapeutic regimes as the salvage therapy between 2014 and 2017. Each cycle consisted of irinotecan $\left(50 \mathrm{mg} / \mathrm{m}^{2} /\right.$ day intravenously for $\left.90 \mathrm{mins}\right)$ on days $1-5$ and temozolomide $\left(100 \mathrm{mg} / \mathrm{m}^{2} /\right.$ day orally 1 $\mathrm{h}$ before irinotecan) on days $1-5$, and vincristine $(1.5 \mathrm{mg} /$ $\mathrm{m}^{2} /$ day intravenously) on day 1 . The schedule is described in Table S1. Atropine $(0.01 \mathrm{mg} / \mathrm{kg})$ was administered $0.5 \mathrm{~h}$ prior to irinotecan as a prophylactic treatment for adverse gastrointestinal effects. Loperamide was administered orally at a dosage of $0.08-0.24 \mathrm{mg} / \mathrm{kg}$ body weight daily in two or three doses when the patients experienced diarrhea following an infusion with irinotecan. ${ }^{23}$ Patients who experienced severe diarrhea (grade III/IV) during the first course of VIT therapy would receive cefixime $(1.5-6 \mathrm{mg} / \mathrm{kg})$ orally as prophylactic treatment prior to each course of following VIT therapies. This study was approved by the SYSUCC Institutional Review Board and the Research Ethics Committee (IRB: B2020-313-01). The confidentiality and compliance of the patient data were conducted in accordance with the World Medical Association Declaration of Helsinki. Since this was a retrospective study and the exemption of informed consent does not harm the rights and health of the subjects, informed consent was waived by SYSUCC Institutional Review Board and the Research Ethics Committee.

\section{Irinotecan-Related Toxicities}

Irinotecan-related toxicities (eg, vomiting, diarrhea, abdominal pain, and hematological suppression) were recorded in detail following irinotecan treatment until the subsequent course of chemotherapy. Adverse events were 
assessed using the NCI Common Terminology Criteria for Adverse Events version (CTCAE) 3.0.

\section{Detection of UGTIAI Polymorphisms}

A $3 \mathrm{~mL}$ EDTA peripheral blood sample was collected from each patient. Genomic DNA was isolated from the blood samples using a Qiagen DNA Blood Mini Kit (\# 51104, Qiagen, Germany) according to the manufacturer's protocol. Genotyping of the TATAA box sequence repeat (UGT1A1*28) in the UGT1A1 promoter region and UGT1A1*6 (rs4148323), a SNP also known as $211 \mathrm{G}>$ A or G71R, at exon 1 were determined by sequencing polymerase chain reaction (PCR) amplicons. PCR was performed according to the following conditions: $94^{\circ} \mathrm{C}$ for $5 \mathrm{~min}$, followed by $94^{\circ} \mathrm{C}$ for $30 \mathrm{~s}, 58^{\circ} \mathrm{C}$ for $50 \mathrm{~s}$, $72^{\circ} \mathrm{C}$ for $30 \mathrm{~s}$ for 30 cycles, and finally, $72^{\circ} \mathrm{C} 5$ for min. The PCR amplicons were used for Sanger sequencing. The primers used in this study are listed in Table S2. For the *28 site, the subjects were categorized as three different genotypes depending on the number of TA repeats in the promoter region of the UGT1A1 gene: $6 / 6$ wild type, $6 / 7$ heterozygote, and $7 / 7$ homozygote. For the $* 6$ site, the subjects were grouped into three genotypes: $\mathrm{G} / \mathrm{G}$ wild type, G/A heterozygote, and A/A homozygote.

\section{Follow-Up}

Progression disease (PD), relapse, and death due to any cause were evaluated as events. The overall survival (OS) time was calculated from the date of first VIT therapy to the date of death. The event-free survival (EFS) time was from the date of first VIT therapy to the date of the occurrence of any event, including death, disease progression, second tumor development, or lethal or intolerable side effects. Patients who had not died or experienced other events at the final analysis were censored at the last follow-up in September 2020.

\section{Statistical Analyses}

Statistical analyses were performed using IBM SPSS Statistics 25.0 software (SPSS Inc. Headquarters, Chicago, IL, USA). Deviations from the HardyWeinberg $(\mathrm{H}-\mathrm{W})$ equilibrium of the UGT1A1 $* 6 / * 28$ polymorphism genotypes and the association between categorical variables were tested using a chi-squared test. EFS and OS were analyzed in accordance with the Kaplan-Meier (K-M) method using the patient followup; differences were compared using a log-rank (Mantel Cox) test. The K-M survival curves were plotted using
GraphPad Prism 8.0 (GraphPad Software, San Diego, CA, USA). P values $<0.05$ were considered to be significant. All data in our study have been recorded at SYSUCC for future reference (RDDA2020001730, https://www. researchdata.org.cn).

\section{Results \\ Patient Clinical Characteristics}

The characteristics of the 44 eligible patients who received 203 courses of VIT therapy (median, 4 courses; range: 1-11 courses) are summarized in Table 1 . There were 26 (59.1\%) boys and 18 (40.9\%) girls, and the median age at the initial diagnosis was five years (range: 1-15 years). The median age at the time of the first VIT therapy was six years (range: 1-18years). The most common diagnosis was NB ( $\mathrm{n}=25 ; 56.8 \%)$, and a quarter of the patients were diagnosed as RMS $(\mathrm{n}=11)$. The remaining patients were diagnosed with Wilm's tumor (WM) $(n=4 ; 9.1 \%)$, medulloblastoma (MB) $(\mathrm{n}=2 ; 4.5 \%)$, and desmoplastic small round cell tumor (DSRCT) $(\mathrm{n}=2 ; 4.5 \%)$. Both the UGT1A $1 * 6$ and $* 28$ alleles exhibited $\mathrm{H}-\mathrm{W}$ equilibrium in the studied population (UGT1A1 $* 6$ variant: $\chi^{2}=0.0164$, $P=0.8981 ; \mathrm{UGT} 1 \mathrm{~A} 1 * 28$ variant: $\chi^{2}=0.1855, P=$ $0.6667)$. The frequency of the UGT1A $1 * 6$ wild $(\mathrm{G} / \mathrm{G})$, heterozygous $(\mathrm{G} / \mathrm{A})$, and homozygous $(\mathrm{A} / \mathrm{A})$ genotypes was $31(70.4 \%), 12(27.3 \%)$, and $1(2.3 \%)$, respectively. The majority of patients $(\mathrm{n}=34 ; 77.3 \%)$ carried the UGT1A1*28 wild $(6 / 6)$ genotype, whereas there were 9 $(20.3 \%)$ and $1(2.3 \%)$ patients who carried the heterozygous (6/7), and homozygous (7/7) genotypes, respectively.

\section{Association Between Genetic Polymorphisms and Irinotecan-Related Toxicities}

The prevalence of irinotecan-related toxicities in children with relapsed/refractory solid tumors is presented in Tables 2 and 3. Grade III-IV diarrhea, abdominal pain, and vomiting were observed in $18.2 \%,(\mathrm{n}=37), 3.4 \%$ $(\mathrm{n}=7)$, and $4.9 \%(\mathrm{n}=10)$ courses of VIT therapy, respectively. Diarrhea and abdominal pain that lasted $\geq 4$ days were observed in $18.2 \%(n=37)$ and $10.3 \%(n=21)$ of courses of VIT therapy, respectively. For hematological suppression, grade III-IV leucopenia $(\mathrm{n}=49,24.1 \%)$ and neutropenia $(n=58,28.6 \%)$ occurred more frequently than anemia $(\mathrm{n}=23,11.3 \%)$ and thrombocytopenia $(\mathrm{n}=13$, $6.4 \%$ ). The frequency of grade III-IV toxicities was not significantly different between wild and mutant UGT1A1 
Table I Clinical Characteristics of the Investigated Pediatric Patients with Relapsed/Refractory Solid Tumors $(n=44)$

\begin{tabular}{|c|c|c|}
\hline Characteristics & $\begin{array}{c}\text { No. of Patients } \\
\text { (\%) }\end{array}$ & $\begin{array}{c}\text { No. of VIT Courses } \\
\text { (\%) }\end{array}$ \\
\hline $\begin{array}{l}\text { Age at initial } \\
\text { diagnosis, y } \\
\text { Median } \\
\text { Range }\end{array}$ & $\begin{array}{c}5 \\
1-15\end{array}$ & \\
\hline $\begin{array}{l}\text { Age at first VIT } \\
\text { therapy, y } \\
\text { Median } \\
\text { Range }\end{array}$ & $\begin{array}{c}6 \\
1-18\end{array}$ & \\
\hline $\begin{array}{l}\text { Sex } \\
\text { Boys } \\
\text { Girls }\end{array}$ & $\begin{array}{l}26(59.1) \\
18(40.9)\end{array}$ & $\begin{array}{l}136(67.0) \\
67(33.0)\end{array}$ \\
\hline $\begin{array}{l}\text { Diagnosis } \\
\text { NB } \\
\text { RMS } \\
\text { WM } \\
\text { MB } \\
\text { DSRCT }\end{array}$ & $\begin{array}{l}25(56.8) \\
\text { II }(25.0) \\
4(9.1) \\
2(4.5) \\
2(4.5)\end{array}$ & $\begin{array}{c}144(70.9) \\
39(19.2) \\
10(4.9) \\
4(2.0) \\
6(3.0)\end{array}$ \\
\hline $\begin{array}{l}\text { UGTIAI *6 } \\
\text { 7IGly/Gly } \\
\text { 7IGly/Arg } \\
\text { 7IArg/Arg } \\
\text { MT }\end{array}$ & $\begin{array}{l}31(70.4) \\
12(27.3) \\
1(2.3) \\
13(29.6)\end{array}$ & $\begin{array}{l}150(73.9) \\
48(23.6) \\
5(2.5) \\
53(26.1)\end{array}$ \\
\hline $\begin{array}{l}\text { UGTIAI *28 } \\
(\text { TA)6/(TA)6 } \\
(\text { TA)6/(TA)7 } \\
(\text { TA)7/(TA)7 } \\
\text { MT }\end{array}$ & $\begin{array}{c}34(77.3) \\
9(20.3) \\
1(2.3) \\
10(22.6)\end{array}$ & $\begin{array}{c}157(77.3) \\
38(18.7) \\
8(3.9) \\
46(22.6)\end{array}$ \\
\hline $\begin{array}{l}\text { First event } \\
\text { None } \\
\text { PD } \\
\text { Relapse } \\
\text { Death }\end{array}$ & $\begin{array}{c}8(18.2) \\
17(38.6) \\
9(20.5) \\
10(22.7)\end{array}$ & \\
\hline $\begin{array}{l}\text { Follow-up status } \\
\text { Alive } \\
\text { Death }\end{array}$ & $\begin{array}{l}15(34.1) \\
29(65.9)\end{array}$ & \\
\hline $\begin{array}{l}\text { Follow-up time, m } \\
\text { Median } \\
\text { Range }\end{array}$ & $\begin{array}{c}35 \\
1-78\end{array}$ & \\
\hline
\end{tabular}

Abbreviations: NB, neuroblastoma; RMS, rhabdomyosarcoma; WM, Wilm's tumor; MB, medulloblastoma; PD, progression disease; DSRCT, desmoplastic small round cell tumor; MT, mutant type.

$* 6 / * 28$ genotypes. However, the duration of diarrhea and abdominal pain were correlated with the UGT1A1 *28 polymorphism. The incidence rates of $\geq 4$ days of diarrhea $(22.9 \%$ vs $2.2 \% ; P=0.001)$ and $\geq 4$ days of abdominal
Table 2 Prevalence of CPT-II-Related Toxicities in Children with Relapsed/Refractory Solid Tumors $(n=203)$

\begin{tabular}{|l|c|c|c|}
\hline \multirow{2}{*}{ Toxicity } & \multicolumn{3}{|c|}{ Number of Courses (\%) } \\
\cline { 2 - 4 } & Grade 0 & Grade I-II & Grade III-IV \\
\hline Diarrhea & $97(47.8)$ & $69(34.0)$ & $37(18.2)$ \\
Abdominal pain & $138(68.0)$ & $56(27.6)$ & $7(3.4)$ \\
Vomiting & $17 I(84.2)$ & $22(10.8)$ & $10(4.9)$ \\
Leucopenia & $68(33.5)$ & $86(42.4)$ & $49(24.1)$ \\
Neutropenia & $82(44.4)$ & $63(31.0)$ & $58(28.6)$ \\
Anemia & $4 I(20.2)$ & $139(68.5)$ & $23(11.3)$ \\
Thrombocytopenia & $171(84.2)$ & $19(9.4)$ & $13(6.4)$ \\
\hline
\end{tabular}

Table 3 Duration of Diarrhea and Abdominal Pain in Children with Relapsed/Refractory Solid Tumors $(n=203)$

\begin{tabular}{|l|c|c|c|}
\hline \multirow{2}{*}{ Duration (Day) } & \multicolumn{3}{|c|}{ Number of Courses (\%) } \\
\cline { 2 - 4 } & $\mathbf{0}$ & I-3 & $\geq 4$ \\
\hline Diarrhea & $97(47.8)$ & $69(34.0)$ & $37(18.2)$ \\
Abdominal pain & $138(68.0)$ & $44(21.7)$ & 2 I (I0.3) \\
\hline
\end{tabular}

pain $(12.7 \%$ vs $2.2 \% ; P=0.039)$ were significantly higher in patients with the UGT1A1 *28 wild-type genotype (Table 4).

Next, we dichotomized the toxicity grades as grade 0 versus grade I-IV, and performed a chi-squared test to further evaluate the influence of the UGT1A1 *6/*28 polymorphism on the irinotecan-related toxicities. As shown in Table 5, the results indicated that the UGT1A1*6 polymorphism was correlated with diarrhea and anemia. Patients with the mutant genotype were more pronounced to experience diarrhea $(48.0 \%$ vs $64.2 \% ; P=0.043)$ and anemia $(74.7 \%$ vs $94.3 \%) ; P=$ 0.002). The UGT1A1*28 polymorphism was correlated with abdominal pain, and the patients with a mutant genotype had a significantly lower risk of abdominal pain $(38.2 \%$ vs $10.9 \% ; \mathrm{P}<0.001)$ and tended to have a lower risk of diarrhea (55.4\% vs $41.3 \%)$.

\section{Influence of UGTIAI $* 6 / * 28$ on the Survival of Pediatric Patients with Relapsed/Refractory Solid Tumors}

The median follow-up time was 35 months (range: 1-78 months) for all patients. The first events were observed as PD in 8 patients (18.2\%), relapse in 9 patients (20.5\%), and death in 10 patients $(22 / 7 \%)$. As of the last follow-up on September 2020, 29 patients (65.9\%) died of cancer. 
Table 4 Association Between the UGTIAI *6/*28 Polymorphism and Irinotecan-Related Toxicities (G0-II vs GIII-IV)

\begin{tabular}{|c|c|c|c|c|c|c|c|c|}
\hline \multirow[t]{2}{*}{ Toxicity (GIII-IV) } & \multicolumn{2}{|c|}{ UGTIAI *6 } & \multirow[t]{2}{*}{$\chi^{2}$} & \multirow[t]{2}{*}{$P$} & \multicolumn{2}{|c|}{ UGTIAI $* 28$} & \multirow[t]{2}{*}{$\chi^{2}$} & \multirow[t]{2}{*}{$P$} \\
\hline & WT & MT & & & WT & MT & & \\
\hline Diarrhea & $29(19.3)$ & $8(15.1)$ & 0.472 & 0.492 & $29(18.5)$ & $8(17.4)$ & 0.028 & 0.867 \\
\hline Abdominal pain & $7(4.7)$ & $0(0)$ & 2.562 & 0.109 & $7(4.5)$ & $0(0)$ & 2.124 & 0.145 \\
\hline Vomiting & $6(4.0)$ & $4(7.5)$ & 1.052 & 0.305 & $9(5.7)$ & I (2.2) & 0.962 & 0.327 \\
\hline Leucopenia & $35(23.3)$ & $14(26.4)$ & 0.203 & 0.652 & $4 I(26.1)$ & $8(17.4)$ & 1.478 & 0.224 \\
\hline Neutropenia & $4 \mid(27.3)$ & $17(32.1)$ & 0.432 & $0.5 \mathrm{II}$ & $44(28.0)$ & $14(28.6)$ & 0.101 & 0.750 \\
\hline Anemia & $16(10.7)$ & 7 (I3.2) & 0.252 & 0.616 & $15(9.6)$ & $8(17.4)$ & 2.175 & 0.140 \\
\hline Thrombocytopenia & $7(4.7)$ & $6(11.3)$ & 2.893 & 0.089 & II (7.0) & $2(4.3)$ & 0.420 & 0.517 \\
\hline Duration of diarrhea $(\geq 4 d)$ & $29(19.3)$ & $8(15.1)$ & 0.472 & 0.492 & $36(22.9)$ & I (2.2) & 10.281 & 0.001 \\
\hline Duration of abdominal pain $(\geq 4 d)$ & $16(10.7)$ & $5(9.4)$ & 0.064 & 0.800 & $20(12.7)$ & I (2.2) & 4.282 & 0.039 \\
\hline
\end{tabular}

Table 5 Association Between the UGTIAI *6/*28 Polymorphism and Irinotecan-Related Toxicities (G0 vs GI-IV)

\begin{tabular}{|c|c|c|c|c|c|c|c|c|}
\hline \multirow[t]{2}{*}{ Toxicity (GI-IV) } & \multicolumn{2}{|c|}{ UGTIAI *6 } & \multirow[t]{2}{*}{$\chi^{2}$} & \multirow[t]{2}{*}{$P$} & \multicolumn{2}{|c|}{ UGTIAI $* 28$} & \multirow[t]{2}{*}{$\chi^{2}$} & \multirow[t]{2}{*}{$\boldsymbol{P}$} \\
\hline & WT & MT & & & WT & MT & & \\
\hline Diarrhea & $72(48.0)$ & $34(64.2)$ & 4.094 & 0.043 & $87(55.4)$ & $19(41.3)$ & 2.839 & 0.092 \\
\hline Abdominal pain & $48(32.0)$ & $17(32.1)$ & $<0.001$ & 0.992 & $60(38.2)$ & $5(10.9)$ & 12.228 & $<0.001$ \\
\hline Vomiting & $23(15.3)$ & $9(17.0)$ & 0.080 & 0.777 & $26(16.6)$ & $6(13.0)$ & 0.331 & 0.565 \\
\hline Leucopenia & $100(66.7)$ & $35(66.0)$ & 0.007 & 0.934 & $109(69.4)$ & $26(56.5)$ & 2.660 & 0.103 \\
\hline Neutropenia & 91 (60.7) & 31 (58.5) & 0.077 & $0.78 I$ & $95(60.5)$ & $27(58.7)$ & 0.049 & 0.825 \\
\hline Anemia & $112(74.7)$ & $50(94.3)$ & 9.404 & 0.002 & $121(77.1)$ & 41 (89.1) & 3.221 & 0.073 \\
\hline Thrombocytopenia & $21(14.0)$ & II (20.8) & 1.346 & 0.246 & $21(13.4)$ & II (23.9) & 2.975 & 0.085 \\
\hline
\end{tabular}

The one- to five-year EFS and OS are listed in Table S3. The K-M survival analysis revealed that both $\mathrm{UGT1A1*6}$ and $* 28$ polymorphisms were not significantly associated with the EFS and OS (Figure 1A-D); however, patients with the UGT1A1*6 $(P=0.146)$ or $* 28(P=0.195)$ mutant genotypes tended to have an inferior OS compared to those with the wild-type genotype.

\section{Discussion}

The SNP UGT1A1*6 is a missense polymorphism that leads to a single amino acid change from glycine to arginine (Gly71Arg) at site 71 of the UGT1A1 gene. Such substitution alters the hydrophobicity and secondary structure of the protein. ${ }^{17,24}$ The in vitro studies revealed that the enzymatic activity of the UGT $1 \mathrm{~A} 1 * 6$ variant is approximately one-third of that of the $* 1$ allele. ${ }^{12}$ The UGT1A $1 * 28$ polymorphism results from a change in the number of TA repeats in the TATA box of the UGT1A1 promoter from six wild-type repeats to seven variant repeats. The overall number of TA repeats varies from 5 to 8. UGT1A1 expression in individuals with the variant TA7 allele, especially homozygotes, could be decreased by as much as $70 \%{ }^{5,6,12}$ Therefore, UGT1A1*6 and $* 28$ polymorphisms are theoretically expected to reduce the glucuronidation of SN-38 and could increase the risk of irinotecan-induced toxicities. The association between irinotecan-related toxicities and UGT1A1 genetic polymorphisms in a variety of malignancies have been widely acknowledged and considered for therapeutic recommendations in many countries. ${ }^{25}$ In 2005 , UGT1A1*28 polymorphism genotyping before prescribing irinotecan was recommended by the Food and Drug Administration to reduce irinotecan related toxicities, due to the increased incidence of neutropenia in adult patients with the $7 / 7$ genotype after each 21-day dosage of irinotecan. ${ }^{9,14,26}$ Reduced initial irinotecan dosages should be considered in patients with the UGT1A1*28 genotype. ${ }^{5,16}$ However, controversial results concerning the relevance of this test for predicting irinotecan toxicity have been reported in several studies. ${ }^{16,27}$ The study by Cecchin et al proposed that genotyping a few markers in the UGT1A genes, rather than a single UGT1A $1 * 28$ polymorphism, can significantly improve the predicted outcome of CRC patients, and indicates that the optimal dose reduction in $* 28 / * 28$ patients, which would not compromise its efficacy. ${ }^{16}$ 

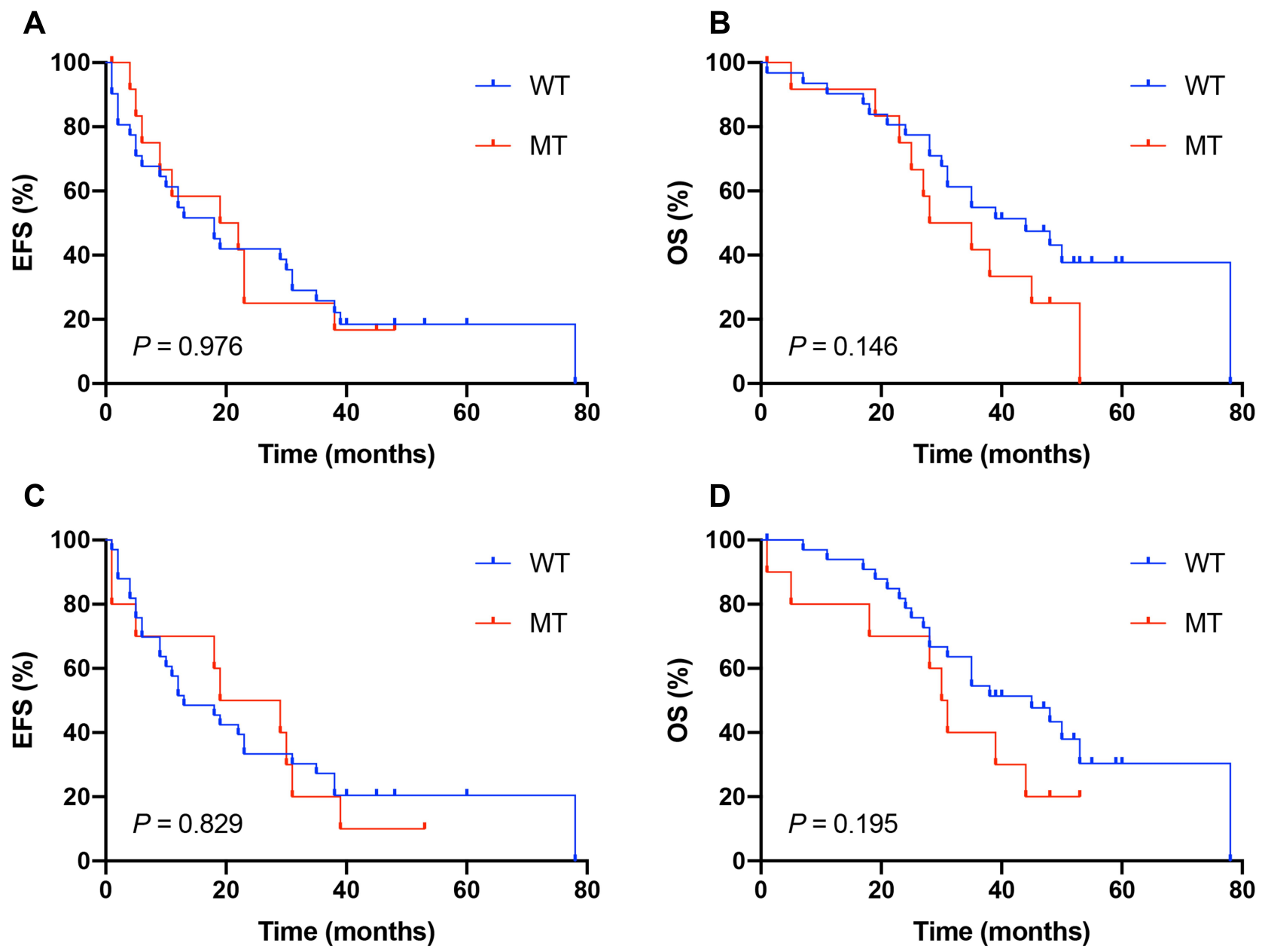

Figure I Kaplan-Meier analysis of event-free survival (EFS) and overall survival (OS) in the pediatric relapsed/refractory solid tumors with genotype of the UGTIAI*6/*28 polymorphisms ( $n=44)$; No significant difference was found in EFS/OS among the different genotypes; WT, wild genotype; MT, mutant genotype. (A) The three-year EFS rate for UGTIAI*6 WT and MT was $25.8 \%$ and $16.7 \%$, Respectively. (B) Patients carrying UGTIAI*6 MT had a non-significantly higher three-year OS rate than those with WT (54.8\% vs $41.7 \%$ ). (C) The three-year EFS rate for UGTIAI*28 WT and MT was $27.3 \%$ and $20.0 \%$, respectively. (D) Patients carrying UGTIAI* 28 MT had a nonsignificantly higher three-year OS rate than those with WT (54.5\% vs $40.0 \%)$.

Numerous studies have investigated the association between UGT1A $1 * 6 / * 28$ polymorphisms and irinotecan toxicities in adult solid tumors, focusing on CRC, lung cancer, and gastric cancer, with inconsistent results. Thus, a series of meta-analyses have been conducted to investigate this topic. Consistent conclusions were made regarding the UGT1A1*6 polymorphism, which could be regarded as a biomarker of irinotecan-related neutropenia and diarrhea, and can particularly benefit Asian cancer patients. ${ }^{4,24,28-33}$ Several of the most recent studies in Asia have experienced similar results. ${ }^{17,34-36}$ The UGT1A1*28 polymorphism has also been reported to be correlated with irinotecan-related diarrhea; however, the conclusions were controversial for neutropenia. The majority of meta-analyses ${ }^{4,24,28-33}$ and two recent studies performed in $\mathrm{Asia}^{35,36}$ concluded that the UGT1A1*28 polymorphism could increase the risk of neutropenia. Campbell et al revealed that such an effect was greater than that of neutropenia in both Asian and Caucasian patients. $^{28}$ Other studies have showed that the UGT1A1*28 polymorphism was not associated with neutropenia or diarrhea and may not be a suitable predictor of irinotecan toxicity. ${ }^{5,30}$ Moreover, a recent Japanese study, which included a total of 651 patients with a wide-range of adult solid tumors and hematopoietic malignancies concluded that UGT1A1 testing could be useful to predict irinotecan-induced adverse reactions, and that UTG1A $1 * 6$ rather than UGT1A $1 * 28$ contributed to the occurrence of adverse drug reactions. ${ }^{25}$ Another recent study from Guangxi Zhuang in China also indicated that UGT1A1*28 was not correlated with irinotecan-related neutropenia in metastatic CRC patients. ${ }^{34}$ 
Compared to adult solid tumors, data is limited regarding the influence of UGT1A $1 * 6 / * 28$ on irinotecan toxicity in pediatric malignancies. Two clinical trials have suggested that the UGT1A1 genotype was not associated with irinotecan-related neutropenia and diarrhea in pediatric refractory solid tumors treated with a low-dose protracted schedule of irinotecan. ${ }^{3,7}$ Many clinical and pharmacogenetic factors, including gender, age, drug administration schedule, and metabolic enzymes, have been reported to be associated with irinotecan toxicity. ${ }^{12,14}$ Therefore, our analysis only enrolled pediatric patients with relapsed/refractory solid tumors who received VIT regimens with irinotecan administrated at a dose of $50 \mathrm{mg} / \mathrm{m}^{2}$ to minimize the impact of cofounding factors on irinotecan toxicity. Previous studies have reported that the frequency of the UGT1A $1 * 28$ polymorphism is $39 \%$ in the white population, whereas that in Asians was only $16 \%,{ }^{37}$ which is similar to the $10 \%$ reported in our study. The mutation rates of UGT1A $1 * 6$ and $* 28$ are similar in the present study. Our findings suggest that both UGT1A $1 * 6$ and $* 28$ polymorphisms were associated with irinotecan-related toxicity. Consistent with previous reports of adult malignancies, pediatric patients with relapsed/refractory solid tumors who carried mutant UGT1A $1 * 6$ genotypes were found to be more likely to experience diarrhea $(P=$ 0.043 ) in our study; however, the UGT1A1*6 polymorphism increased the risk of anemia $(P=0.002)$ rather than neutropenia. In contrast, the UGT1A1*28 polymorphism was not related to bone marrow suppression and interestingly, may play a protective role against diarrhea and abdominal pain. Patients with the mutant UGT1A1*28 genotype were less likely to experience abdominal pain $(P<0.001)$ and tended to have lower risk of diarrhea $(P=0.092)$. Moreover, mutant UGT1A $1 * 28$ genotypes were correlated with a shorter duration of diarrhea $(P=0.001)$ and abdominal pain $(P=0.039)$. All of the irinotecan-related toxicities in our study were manageable with medication. Previous research showed that the influence of UGT1A $1 * 6 / * 28$ polymorphisms on irinotecan toxicity varied by race, region, cancer type, irinotecan dose, and duration of treatment. ${ }^{28,29}$ In addition, the adult dose and schedule of irinotecan varies significantly compared to that of children. This may partially account for the discrepancy between our study and previous research, especially the data pertaining to adults. Also, the sample number in our study was relatively small compared to studies in adult; younger patients (preschool-aged children) added to the difficulty to record the grade of subjective toxicities in the current study. Moreover, the prophylactic treatment of cefixime in patients with previous severe diarrhea may have disguised the influence of UGT1A1 polymorphisms in irinotecan-related diarrhea.

Although the association between UGT1A $1 * 6 / * 28$ and irinotecan toxicity was extensively studied, there is a lack of evidence regarding whether UGT1A $1 * 6 / * 28$ genotyping is of clinical value for predicting survival following irinotecan therapy. Previous observational studies evaluating the influence of UGT $1 \mathrm{~A} 1 * 6 / * 28$ on survival have revealed contradictory results. Reviews suggest that the UGT1A $1 * 28$ polymorphism was not associated with any changes to the objective response rate, progression-free survival (PFS), or overall survival (OS) following irinotecan treatment. ${ }^{4,38}$ A recent study also found that neither UGT1A $1 * 6$ nor $* 28$ had an impact on treatment efficacy and PFS in metastatic CRC; ${ }^{34}$ however, Matsuoka et al suggested that UGT1A1 polymorphisms were significantly related with longer PFS in uterine cervical cancer. ${ }^{39}$ Similarly, the findings by Henriksen et al revealed that UGT1A1*28 improved the PFS and OS in metastatic renal cell carcinoma. $^{40}$ In contrast, the study by Yamaguchi et al confirmed that UGT1A1*6 and $* 28$ were shown to be related to a shorter OS in advanced gastric cancer when irinotecan monotherapy was used as a third-line treatment. ${ }^{41}$ In the present study, the $\mathrm{K}$ $\mathrm{M}$ survival analysis revealed that patients with mutant UGT1A1*6 $(P=0.146)$ or $* 28(P=0.195)$ genotypes had a non-significant inferior OS compared to those with the wild genotype.

We are aware of several limitations associated with this study. The major limitation is the retrospective nature. Since not all patients were willing to be genotyped for the UGT1A1 $6 * / * 28$ polymorphism, there was a small sample number included in this study, and this was a single center study. Additionally, irinotecan metabolism is influenced by both UGT1A1*6/*28, as well as several other genetic polymorphisms, including CYP3A4/5, DPYD, CES2, SLCO1B1, ABCB1, and ABCC2. ${ }^{14,18,35}$ Therefore, taking only UGT1A1*6/*28 into consideration may not be sufficiently convincing to explore the relationship with irinotecan toxicities. In conclusion, UGT1A $1 * 6$ and $* 28$ polymorphisms may represent biomarkers of irinotecan toxicities but not to the extent of severe toxicities (grade III-IV). In addition, UGT1A1*6 and *28 genotyping may have use for predicting survival following VIT regimens in pediatric patients with relapsed/refractory solid tumors. Nevertheless, further prospective multicenter studies are required to validate the predictive significance of these associations. 


\section{Abbreviations}

UGT1A1, UDP-glycosyltransferase 1 polypeptide A1; VIT regimen, irinotecan, vincristine, temozolomide; CRC, colorectal cancer; NB, neuroblastoma; RMS, rhabdomyosarcoma; SNP, single-nucleotide polymorphism; SYSUCC, Sun Yat-sen University Cancer Center; CTCAE, NCI Common Terminology Criteria for Adverse Events version; PD, progression disease; OS, overall survival; EFS, event-free survival; H-W, Hardy-Weinberg; K-M, Kaplan-Meier; W-M, Wilm's tumor; MB, medulloblastoma; DSRCT, desmoplastic small round cell tumor; PFS, progression-free survival.

\section{Disclosure}

The authors report no conflicts of interest in this work.

\section{References}

1. Modak S, Kushner B, Basu E, Roberts S, Cheung N. Combination of bevacizumab, irinotecan, and temozolomide for refractory or relapsed neuroblastoma: results of a Phase II study. Pediatr Blood Cancer. 2017;64(8):e26448. doi:10.1002/pbc.26448

2. Jinno H, Tanaka-Kagawa T, Hanioka N. Glucuronidation of 7-ethyl10-hydroxycamptothecin (SN-38), an active metabolite of irinotecan (CPT-11), by human UGT1A1 variants, G71R, P229Q, and Y486D. Drug Metab Dispos. 2003;31(1):108-113. doi:10.1124/ dmd.31.1.108

3. Bomgaars L, Bernstein M, Krailo M, et al. Phase II trial of irinotecan in children with refractory solid tumors: a Children's Oncology Group Study. J Clin Oncol. 2007;25(29):4622-4627. doi:10.1200/ JCO.2007.11.6103

4. Campbell JM, Stephenson MD, Bateman E, Peters MD, Keefe DM, Bowen JM. Irinotecan-induced toxicity pharmacogenetics: an umbrella review of systematic reviews and meta-analyses. Pharmacogenomics J. 2017;17(1):21-28. doi:10.10 38/tpj.2016.58

5. Stewart C, Panetta J, O'Shaughnessy M, et al. UGT1A1 promoter genotype correlates with SN-38 pharmacokinetics, but not severe toxicity in patients receiving low-dose irinotecan. J Clin Oncol. 2007;25(18):2594-2600. doi:10.1200/JCO.2006.10.2301

6. Nagar S, Blanchard R. Pharmacogenetics of uridine diphosphoglucuronosyltransferase (UGT) 1A family members and its role in patient response to irinotecan. Drug Metab Rev. 2006;38 (3):393-409. doi:10.1080/03602530600739835

7. McGregor L, Spunt S, Furman W, et al. Phase 1 study of oxaliplatin and irinotecan in pediatric patients with refractory solid tumors: a children's oncology group study. Cancer. 2009;115(8):1765-1775. doi:10.1002/cncr.24175

8. Federico S, Pappo A, Sahr N, et al. A Phase I trial of talazoparib and irinotecan with and without temozolomide in children and young adults with recurrent or refractory solid malignancies. Eur J Cancer. 2020;137:204-213. doi:10.1016/j.ejca.2020.06.014

9. Vassal G, Doz F, Frappaz D, et al. A phase I study of irinotecan as a 3-week schedule in children with refractory or recurrent solid tumors. J Clin Oncol. 2003;21(20):3844-3852. doi:10.1200/JCO. 2003.08.175

10. Blaney S, Berg S, Pratt C, et al. A phase I study of irinotecan in pediatric patients: a pediatric oncology group study. Clin Cancer Res. 2001;7(1):32-37.
11. Mody R, Naranjo A, Van Ryn C, et al. Irinotecan-temozolomide with temsirolimus or dinutuximab in children with refractory or relapsed neuroblastoma (COG ANBL1221): an open-label, randomised, Phase 2 trial. Lancet Oncol. 2017;18(7):946-957. doi:10.1016/S14702045(17)30355-8

12. Kweekel D, Guchelaar H, Gelderblom H. Clinical and pharmacogenetic factors associated with irinotecan toxicity. Cancer Treat Rev. 2008;34(7):656-669. doi:10.1016/j.ctrv.2008.05.002

13. Nakayama G, Tanaka C, Uehara K, et al. The impact of dose/time modification in irinotecan- and oxaliplatin-based chemotherapies on outcomes in metastatic colorectal cancer. Cancer Chemother Pharmacol. 2014;73(4):847-855. doi:10.1007/s00280-014-2416-x

14. Ahowesso C, Piccolo E, Li XM, et al. Relations between strain and gender dependencies of irinotecan toxicity and UGT1A1, CES2 and TOP1 expressions in mice. Toxicol Lett. 2010;192(3):395-401. doi:10.1016/j.toxlet.2009.11.017

15. Gupta E, Lestingi T, Mick R, Ramirez J, Vokes E, Ratain M. Metabolic fate of irinotecan in humans: correlation of glucuronidation with diarrhea. Cancer Res. 1994;54(14):3723-3725.

16. Cecchin E, Innocenti F, D'Andrea M, et al. Predictive role of the UGT1A1, UGT1A7, and UGT1A9 genetic variants and their haplotypes on the outcome of metastatic colorectal cancer patients treated with fluorouracil, leucovorin, and irinotecan. J Clin Oncol. 2009;27 (15):2457-2465. doi:10.1200/JCO.2008.19.0314

17. Zhu X, Ma R, Ma X, Yang G. Association of UGT1A1*6 polymorphism with irinotecan-based chemotherapy reaction in colorectal cancer patients: a systematic review and a meta-analysis. Biosci Rep. 2020;40. doi:10.1042/BSR20200576

18. Innocenti F, Undevia SD, Rosner GL, et al. Irinotecan (CPT-11) pharmacokinetics (PK) and neutropenia: interaction among UGT1A1 and transporter genes. J Clin Oncol. 2020.

19. Wasserman E, Myara A, Lokiec F, et al. Severe CPT-11 toxicity in patients with Gilbert's syndrome: two case reports. Ann Oncol. 1997;8(10):1049-1051. doi:10.1023/A:1008261821434

20. Xie F-W, Yh Y-H, Wang W, et al. Influence of UGT1A1 gene methylation level in colorectal cancer cells on the sensitivity of the chemotherapy drug CPT-11. Biomed Pharmacother. 2014;68 (7):825-831. doi:10.1016/j.biopha.2014.08.012

21. Font A, Salazar R, Maurel J. Cisplatin plus weekly CPT-11/docetaxel in advanced esophagogastric cancer: a phase I study with pharmacogenetic assessment of XPD, XRCC3 and UGT1A1 polymorphisms. Cancer Chemother Pharmacol. 2008;62(6):1075-1083. doi:10.1007/ s00280-008-0700-3

22. Tobin PJ, Beale P, Noney L, et al. A pilot study on the safety of combining chrysin, a non-absorbable inducer of UGT1A1, and irinotecan (CPT-11) to treat metastatic colorectal cancer. Cancer Chemother Pharmacol. 2006;57(3):309-316. doi:10.1007/s00280005-0053-0

23. Buts J, Petit B, de Meyer R. Letter: loperamide in treatment of persistent diarrhoea in children. Br Med J. 1975;3(5986):766-767. doi:10.1136/bmj.3.5986.766-d

24. Han FF, Guo CL, Yu D, et al. Associations between UGT1A1*6 or UGT1A $1 * 6 / * 28$ polymorphisms and irinotecan-induced neutropenia in Asian cancer patients. Cancer Chemother Pharmacol. 2014.

25. Hikino K, Ozeki T, Koido M, et al. Comparison of effects of UGT1A1*6 and UGT1A $1 * 28$ on irinotecan-induced adverse reactions in the Japanese population: analysis of the Biobank Japan Project. J Hum Genet. 2019;64(12):1195-1202. doi:10.1038/s10038-019-0677-2

26. Ramchandani R, Wang Y, Booth B, et al. The role of SN-38 exposure, UGT1A1*28 polymorphism, and baseline bilirubin level in predicting severe irinotecan toxicity. J Clin Pharmacol. 2007;47 (1):78-86.

27. Lankisch T, Schulz C, Zwingers T, et al. Gilbert's Syndrome and irinotecan toxicity: combination with UDP-glucuronosyltransferase 1A7 variants increases risk. Cancer Epidemiol, Biomarkers Prev. 2008;17(3):695-701. 
28. Yang Y, Zhou M, Hu M, et al. UGT1A1*6 and UGT1A1*28 polymorphisms are correlated with irinotecan-induced toxicity: a metaanalysis. Asia Pac J Clin Oncol. 2018;14(5):e479-e489. doi:10.1111/ ajco. 13028

29. Zhang X, Yin J, Zhang J, Kong S, Zhang H, Chen X. UGT1A1*6 polymorphisms are correlated with irinotecan-induced neutropenia: a systematic review and meta-analysis. Cancer Chemother Pharmacol. 2017;80(1):135-149. doi:10.1007/s00280-017-3344-3

30. Chen X, Liu L, Guo Z, et al. UGT1A1 polymorphisms with irinotecan-induced toxicities and treatment outcome in Asians with Lung Cancer: a meta-analysis. Cancer Chemother Pharmacol. 2017;79(6):1109-1117. doi:10.1007/s00280-017-3306-9

31. Liu X, Lu J, Duan W, et al. Predictive value of UGT1A1*28 polymorphism in irinotecan-based chemotherapy. J Cancer. 2017;8 (4):691-703. doi:10.7150/jca.17210

32. Cheng $\mathrm{L}$, Li M, Hu J, et al. UGT1A1*6 polymorphisms are correlated with irinotecan-induced toxicity: a system review and metaanalysis in Asians. Cancer Chemother Pharmacol. 2014;73(3):5 51-560. doi:10.1007/s00280-014-2382-3

33. Chen $\mathrm{Y}, \mathrm{Hu} \mathrm{F}, \mathrm{Li} \mathrm{C}$, et al. The association of UGT1A1*6 and UGT1A1*28 with irinotecan-induced neutropenia in Asians: a metaanalysis. Biomarkers. 2014;19(1):56-62. doi:10.3109/1354750X.20 13.867534

34. Chen S, Hua L, Feng C, et al. Correlation between UGT1A1 gene polymorphism and irinotecan chemotherapy in metastatic colorectal cancer: a study from Guangxi Zhuang. BMC Gastroenterol. 2020;20 (1):96. doi:10.1186/s12876-020-01227-w

35. Atasilp C, Chansriwong P, Sirachainan E, et al. Effect of drug metabolizing enzymes and transporters in Thai colorectal cancer patients treated with irinotecan-based chemotherapy. Sci Rep. 2020;10(1):13486. doi:10.1038/s41598-020-70351-0
36. Ma X, Han S, Liu Y, Liu J, Fang J, Zhang Y. Pharmacogenetic impact of UGT1A1 polymorphisms on pulmonary neuroendocrine tumours treated with metronomic irinotecan-based chemotherapy in Chinese populations. J Pharm Pharmacol. 2020;72:1528-1535. doi:10.1111/ jphp. 13333

37. Zhu J, Liu A, Sun X, et al. UGT1A1Multicenter, randomized, Phase III trial of neoadjuvant chemoradiation with capecitabine and irinotecan guided by status in patients with locally advanced rectal cancer. J Clin Oncol. 2020;38:JCO2001932. doi:10.1200/JCO.20.01932

38. Dias M, Pignon J, Karapetis C, et al. The effect of the UGT1A1*28 allele on survival after irinotecan-based chemotherapy: a collaborative meta-analysis. Pharmacogenomics J. 2014;14(5):424 431. doi:10.10 38/tpj.2014.16

39. Matsuoka H, Murakami R, Abiko K, et al. UGT1A1 polymorphism has a prognostic effect in patients with stage IB or II uterine cervical cancer and one or no metastatic pelvic nodes receiving irinotecan chemotherapy: a retrospective study. BMC Cancer. 2020;20(1):729. doi:10.1186/s12885-020-07225-1

40. Henriksen J, Bøttger P, Hermansen C, et al. Pazopanib-induced liver toxicity in patients with metastatic renal cell carcinoma: effect of UGT1A1 polymorphism on pazopanib dose reduction, safety, and patient outcomes. Clin Genitourin Cancer. 2020;18(1):62-68.e62. doi:10.1016/j.clgc.2019.09.013

41. Yamaguchi T, Iwasa S, Shoji H, et al. Association between UGT1A1 gene polymorphism and safety and efficacy of irinotecan monotherapy as the third-line treatment for advanced gastric cancer. Gastric Cancer. 2019;22(4):778-784. doi:10.1007/s10120-018-00917-5
Pharmacogenomics and Personalized Medicine

\section{Publish your work in this journal}

Pharmacogenomics and Personalized Medicine is an international, peer-reviewed, open access journal characterizing the influence of genotype on pharmacology leading to the development of personalized treatment programs and individualized drug selection for improved safety, efficacy and sustainability. This journal is indexed on the American Chemical Society's Chemical Abstracts Service (CAS). The manuscript management system is completely online and includes a very quick and fair peer-review system, which is all easy to use. Visit http://www.dovepress.com/testimonials.php to read real quotes from published authors. 